\section{Receiving Credit When Not an Author: Documenting Acknowledgments for Research Teams}

\section{TO THE EDITOR:}

In the June 2020 issue of Family Medicine, only two brief reports ${ }^{1,2}$ list individuals in the Acknowledgments section as contributing to the submission. Rules of authorship dictate who gets writing credit, but few journals specify how the acknowledgment section should be used. Published research studies represent the work of not only the authors, but also larger research teams and support staff. This is particularly relevant in family medicine residencies and in practice-based research networks (PBRN). Some individuals may have played a critical role in the execution of a study but were not involved in manuscript development. In residencies, this frequently happens because residents contribute to a project and then graduate residency before the study enters the writing phase.

The use of acknowledgments varies across research teams, academic institutions, and journals. The North American Primary Care Research Group PBRN, which provides mentoring and networking to researchers, gives guidance on authorship in article publication and calls for the use of acknowledgments when the level of contribution to a project does not meet authorship guidelines. ${ }^{3}$ Our own PBRN (Military Primary Care Research Network), whose mission is to educate and train investigators, aims to give recognition to all of the learners and team members who were critical in the success of research projects. Research takes a team and none of us can do this alone. We know that every resident and every research associate who has drafted an initial institutional review board protocol, recruited patients, entered data, or proofread our work has contributed to our success. It is imperative as principal investigators and team authors to acknowledge the role of these team members when we are sharing our research study with our peers. A major limitation of this recognition process is that these mentions are not searchable or curated similar to publication authorship. This can make it difficult for research support team members to build an identifiable body of work and be recognized for their role in research.
Although acknowledgments will not have the same weight as authorship, it demonstrates experience and general understanding of research processes. We encourage our research team members to dedicate a section of their curriculum vitae (CV) to document publications on which they've been acknowledged but not met the qualifications to receive authorship. Particularly for young investigators, such as medical students, residents, or junior faculty, listing publications in which they are acknowledged provides evidence of research experience not listed elsewhere on a CV. This gives young investigators a curated list to demonstrate their participation in research and provides a framework for explaining their role in successful projects. We also recommend that they include published articles in which they've been acknowledged in their educator's portfolio. For those drafting a traditional resume, a "Research and Skills" section that links skills to the paper in which they were acknowledged could provide specific evidence of experience.

Understanding not only the qualifications of authorship but also the critical efforts of team members is a lesson we aim to teach and model for our network's physician investigators. We look forward to hearing how other family medicine educators acknowledge team members in their work.

DISCLAIMER: The views expressed in this publication represent those of the authors and do not reflect the official position of the Henry M. Jackson Foundation, the Uniformed Services University of the Health Sciences, the US Government, or the Department of Defense at large. doi: 10.22454/FamMed.2020.953995

\section{Lauren A. Cafferty, MA}

Jeremy T. Jackson, BA

Henry M. Jackson Foundation;

Military Primary Care Research Network,

Uniformed Services University of the Health Sciences,

Department of Family Medicine,

Bethesda, MD

\section{References}

1. Wilhoit-Reeves SB, Sisler LAG, Aymes SE, Ortiz-Pujols SM, Porterfield DS, Goldstein AO. Building a viable weight management program in a patient-centered medical home. Fam Med. 2020;52(6):427-431.

2. Barzin A, Seybold OC, Page C. Integrating an urgent care clinic into an academic family medicine practice. Fam Med. 2020;52(6):440-443.

3. North American Primary Care Research Group. PBRN Research Good Practices (PRGP). 2014. https://www.napcrg. org/media/1196/prgp-2014-09-29.pdf. Accessed June 5, 2020. 